\title{
A Prospective Comparison of Three Strategies for Evaluating Blood Loss in Transurethral Resection of the Prostate
}

\author{
Xiaojuan Yuan, Wei Yu, Ronghua Wu, Longkun Li, and Fan He \\ The Department of Urology, Second Affiliated Hospital, Army Medical University, Chongqing 400037, China \\ Correspondence should be addressed to Fan He; hefan_99@sina.com \\ Xiaojuan Yuan and Wei Yu contributed equally to this work.
}

Received 17 September 2020; Revised 5 February 2021; Accepted 19 March 2021; Published 7 April 2021

Academic Editor: Hossein Tezval

Copyright (C) 2021 Xiaojuan Yuan et al. This is an open access article distributed under the Creative Commons Attribution License, which permits unrestricted use, distribution, and reproduction in any medium, provided the original work is properly cited.

\begin{abstract}
Objective. The aim of the current investigation is to develop a new strategy for evaluating blood loss in the process of transurethral resection of the prostate (TURP). Methods. 318 patients diagnosed with benign prostatic hyperplasia (BPH) that need TURP were enrolled in this study. Hospitalization information including age, height, weight, surgery time, prostate volume, hemoglobin (Hb) concentration, hematocrit (HCT) percentage, and red blood cell count (RBC) was evaluated for each patient. All statistical analysis drawing were conducted using R software. Results. Three methods were employed for calculating blood loss in TURP. Results from a new method display 0 missing value and got higher confidence ( 0 of 318 , Poisson distribution, $P<0.001)$ compared with blood loss calculated with hemoglobin concentration (20.44\%) and hematocrit percentage (19.18\%). Also, the new method demonstrated narrow range $(0.03 \sim 270.03 \mathrm{ml})$ and approximate normal distribution compared with blood loss calculated with hemoglobin concentration and hematocrit percentage. More importantly, the new method explained positive correlation with prostate volume $\left(R^{2}=0.138, P<0.001\right)$ and also surgery lasting time $\left(R^{2}=0.193, P<0.001\right)$. Conclusion. Methods developed for calculating blood loss in TURP in the current study displayed more accurate and reasonable evaluation of bleeding, which can guide the transfusion blood for patients.
\end{abstract}

\section{Introduction}

Transurethral resection of the prostate (TURP) is the most common surgery for benign prostatic hyperplasia (BPH) [1]. Despite its minimally invasive operation, bleeding still shows a common morbidity within and after surgery [2]. Some severe bleeding even requires blood transfusion and influenced the hospitalization and hospital resources [3]. Although improvement of surgical skills, anesthesia, and pharmacologic agents can reduce bleeding rate, transfusion rates during TURP were reported to be as high as $20 \%$ and have no tendency to fall $[4,5]$. Therefore, accurate and reasonable assessment of blood volume in the process of TURP is very important for patient's rehabilitation.

Rapidly and accurately evaluating blood loss during surgery, strictly controlling bleeding, and effectively measuring blood volume are an important part of the perfect operation and good at postoperative rehabilitation for patients [6]. There are no reliable method and literature report to judge the amount of blood loss in the process of TURP [7]. The main strategies to measure intraoperative blood loss in operating rooms are estimation based on the amount of blood absorbed by gauze and estimation based on the induced drainage. All of the above are estimated by observation, with large bias, errors, and low accuracy. In clinical lab, several methods have been referred for evaluating bleeding. Ekengren and Hahn [8], for the first time, used a HemoCue photometer analyzing low-concentration hemoglobin $(\mathrm{Hb})$, which verified to be more accurate. Some scholars [9] used the urine-strip method to measure the intraoperative blood loss in 11 patients who underwent TURP, and the results indicate that the urine-strip and spectrophotometer method were found to be highly correlated. In 2010, Descazeaud et al. [10] used the ${ }^{51} \mathrm{Cr}$ isotope labeling method to determine 
the amount of TURP bleeding. The ${ }^{51} \mathrm{Cr}$ labeling method was used 1 day before and 3 days after the surgery. The results showed that the average RBC loss was $209 \mathrm{ml}$ and the amount of bleeding was $507 \mathrm{ml}$. Univariate analyses showed that prostate volume, resected glandular tissue weight, preoperative RCV, and operative time were significantly associated with RBC loss. However, these methods endured costly, time-consuming character.

In the current investigation, our group develops a new method for evaluating bleeding in TURP and also compared with previous methods measured with hemoglobin concentration and hematocrit percentage. The new method demonstrated convenience and is fast and economic for clinical application.

\section{Materials and Methods}

2.1. Patients. 318 patient diagnosed with $\mathrm{BPH}$ that need TURP in the Second Affiliated Hospital of Army Medical University during the period from June 2018 to April 2019 were enrolled in the current investigation. Patient inclusion criteria included (1) patients diagnosed with $\mathrm{BPH}$ and that need surgery according to the EAU guideline [11], (2) patients with normal coagulation function; and (3) patients with voluntary participation in the study and that signed informed consent. This study was conducted with the approval of the medical ethics committee of the Second Affiliated Hospital, Army Medical University. WHO dehydration assessment tool was employed to perform hydration status before and after surgery in the current investigation. The blood was taken before surgery anesthesia based on patients' consent. No presurgery infusion was done before blood collection. Hospitalization information including age, height, weight, surgery time, prostate volume, hemoglobin ( $\mathrm{Hb})$ concentration, hematocrit (HCT) percentage, and red blood cell count (RBC) was evaluated for each patient.

2.2. Blood Loss Measurement. In the current investigation, three methods for evaluating blood loss were compared with each other. Methods using hemoglobin concentration (formula (2)) and hematocrit percentage (formula (3)) were performed as previously described and widely used in clinical practice. A new strategy (formula (1)) for evaluating blood loss was reported firstly in the current study. Briefly, $2 \mathrm{ml}$ blood was collected from each patient before TURP and was diluted in $3000 \mathrm{ml}$ saline and the amount of red blood cell (cell/ml) was measured for standardization. In addition, within 2 hours after surgery, the amount of red blood cell was calculated in the saline flush. In addition, the amount of red blood cell was calculated by using routine urine analysis. According to the red blood cell measurement before and after surgery, we calculated the blood loss in the process of TURP by the following formula:

Formula 1 : calculated blood loss $(\mathrm{ml})=\mathrm{RBC}_{\mathrm{after}} / \mathrm{RBC}_{\text {before }} \times$ saline $_{\text {after }} / 3000 \times 2$,

where $\mathrm{RBC}_{\text {before }}$ is preoperative red blood cell in saline $($ cell $/ \mu l), \mathrm{RBC}_{\mathrm{after}}$ is postoperative red blood cell in saline
TABLE 1: Clinical characteristic of 318 samples including in current evaluation.

\begin{tabular}{lcc}
\hline & Range (min max) & Mean \pm SD \\
\hline Age (year) & $48 \sim 92$ & $70.58 \pm 8.51$ \\
Height (m) & $1.37 \sim 1.8$ & $1.62 \pm 0.06$ \\
Weight (kg) & $26 \sim 100.4$ & $62.26 \pm 10.4$ \\
BMI & & $23.65 \pm 3.49$ \\
Surgery_time (min) & $10 \sim 85$ & $43.06 \pm 19.32$ \\
Volume (ml) & $20 \sim 79.8$ & $40.76 \pm 15.19$ \\
Hb_before (g/l) & $66 \sim 171$ & $130.63 \pm 15.99$ \\
Hb_after (g/l) & $63 \sim 166$ & $124.91 \pm 16.03$ \\
Hb_count (ml) & $0 \sim 2160$ & $269.18 \pm 288.59$ \\
HCT_before (\%) & $16.1 \sim 52.1$ & $39.45 \pm 4.75$ \\
HCT_after (\%) & $11.9 \sim 50.9$ & $37.7 \pm 4.73$ \\
HCT_count (ml) & $0 \sim 2079$ & $219.64 \pm 246.5$ \\
Newmethod_count (ml) & $0.03 \sim 270.03$ & $23.39 \pm 32.38$ \\
\hline
\end{tabular}

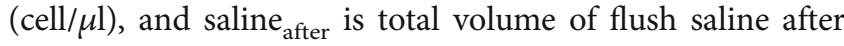
surgery.

Formula 2 : calculated blood loss $(\mathrm{ml})=\left(\mathrm{Hb}_{\text {before }}-\mathrm{Hb}_{\mathrm{after}}\right) \times 40$,

where $\mathrm{Hb}_{\text {before }}$ is preoperative hemoglobin concentration $(\mathrm{g} / \mathrm{l}), \mathrm{Hb}_{\mathrm{after}}$ is postoperative hemoglobin concentration $(\mathrm{g} / \mathrm{l})$, and $40(\mathrm{ml})$ is a value assuming in normal adult (70 kg weight, $5000 \mathrm{ml}$ volume blood, $12.5 \mathrm{~g} / \mathrm{l}$ hemoglobin) hemoglobin loss $1 \mathrm{~g} / \mathrm{l}$ equals $40 \mathrm{ml}$ blood loss (see reference [12]).

Formula 3 : calculated blood loss $(\mathrm{ml})=\left(\mathrm{HCT}_{\text {before }}-\mathrm{HCT}_{\text {after }}\right) / \mathrm{HCT}_{\text {before }} \times 0.07 \times 1000$,

where $\mathrm{HCT}_{\text {before }}$ is preoperative hematocrit percentage (\%) and $\mathrm{HCT}_{\text {after }}$ is postoperative hematocrit percentage (\%) (see reference [13]).

2.3. Statistical Analysis. Clinical characteristics were demonstrated as range (min $\sim \max$ value) and mean \pm SD (standard deviation). Poisson distribution was used for comparing rate. All statistical analysis drawings were conducted using R software (http://www.R-project.org/).

\section{Results}

3.1. Demographic Characters. 318 male patients with $\mathrm{BPH}$ receiving TURP were enrolled in current research. All clinical characters are displayed in Table 1. Age range was from 48 92 years old; the average (mean) and standard deviation (SD) of BMI is $23.65 \pm 3.49$. In addition, the range of surgery lasting time shows from 10 to 85 minutes, and prostate volume is $20 \sim 79.8 \mathrm{ml}$. Pearson correlation was calculated among clinical characters (Figure 1). The results showed that age was 


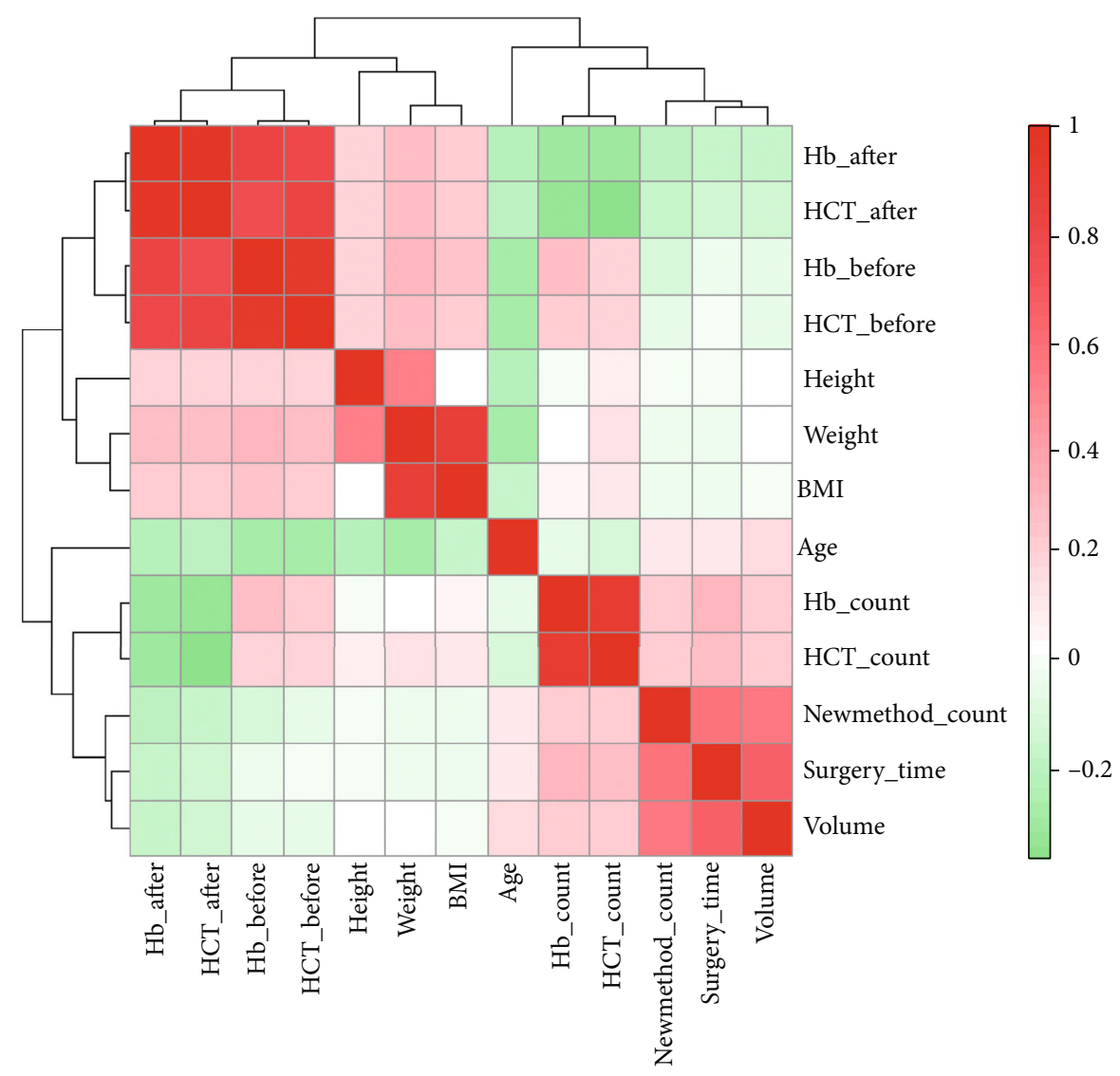

FIGURE 1: Heat map plot of Pearson correlation between clinical characterizations including age, height, weight, BMI, prostate volume, and surgery_time and parameters for evaluating blood volume in the surgery of TURP including Hb_before (hemoglobin value before surgery), Hb_after (hemoglobin value after surgery), HCT_before (hematocrit percent before surgery), HCT_after (hematocrit percent after surgery), $\mathrm{Hb}$ _count (blood volume evaluation according to hemoglobin value), HCT_count (blood volume evaluation according to hematocrit percent), and Newmethod_count (blood volume evaluation according to formula in methods).

negatively correlated with height, weight, hemoglobin concentration, and hematocrit percentage, which was consistent with the actual situation. More importantly, surgery lasting time, prostate volume, and blood loss volume (calculated with formula (1)) were found positively correlation with each other.

3.2. Three Strategies for Evaluating Blood Loss. Three methods were employed for calculating blood loss (see Materials and Methods). The results show that blood loss calculated with formula (1) demonstrated a narrow range from 0.03 to $270.03 \mathrm{ml}$ compared with blood loss calculated with formula (2) (range, 0 2160 ml) and formula (3) (range, 0 2079 ml) (Figure 2(a)). In addition, blood loss volume calculated with hemoglobin concentration and hematocrit percentage has $20.44 \%$ (65 of 318 ) and $19.18 \%$ (61 of 318 ) of 0 value, which was higher than blood loss calculated with formula (1) ( 0 of 318, Poisson distribution, $P<0.001)$. Distribution of blood loss volume was drawn with histogram with density curve fitting, and the results dedicated that the method calculated with formula (1) got a narrow range and approximate normal distribution (Figure 2(b)) compared with skewed distribution of methods calculated with formula (2) (Figure 2(c)) and formula (3) (Figure 2(d)).
3.3. Correlation with Blood Loss, Prostate Volume, and Surgery Lasting Time. Except a more accurate evaluation of blood loss, the method calculated with formula (1) demonstrated positive correlation with prostate volume (Figure 3(a), $R^{2}=0.138, P<0.001$ ) and also surgery lasting time (Figure $3(\mathrm{~b}), R^{2}=0.193, P<0.001$ ). Conversely, we did not find any correlation between blood loss volumes calculated with formula (2) or formula (3) with prostate volume and surgery lasting time (Figures 3(a) and 3(b)).

\section{Discussion}

Based on the important role of evaluating blood loss in the surgery of TURP, the current study describes a method using red cell count (RBC) in flush saline as a reference. Actually, the new method was more accurate for evaluating bleeding and specially displayed better in counting range, variance, fitting curve, and also correlation with prostate volume and surgery lasting time compared with traditional evaluating methods. The advantage of the current method showed in several ways.

In the process of evaluating blood loss, formula (2) and formula (3) get results lesser or equal to zero, and this is because postoperative hemoglobin concentration or 


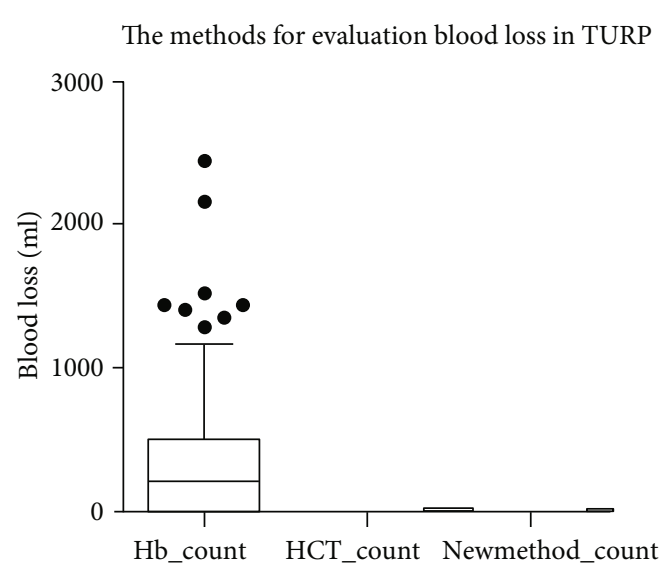

(a)

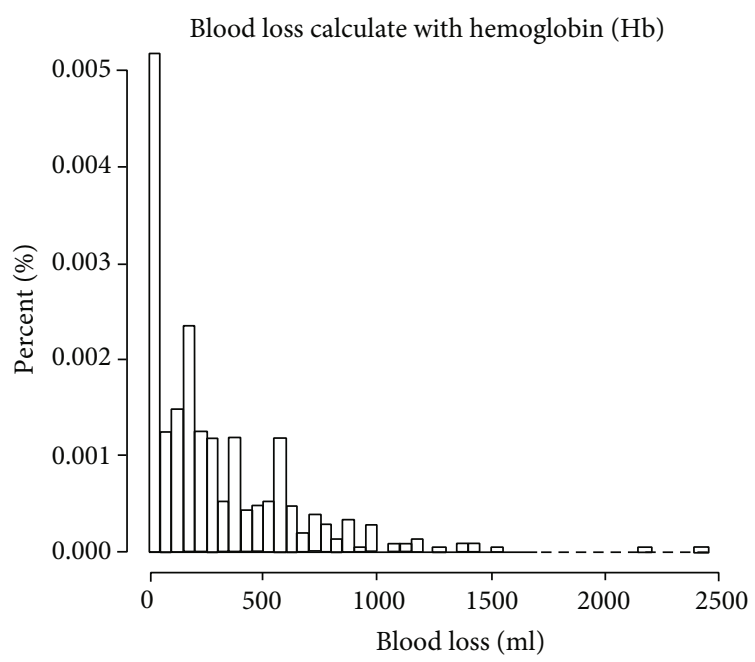

(c)

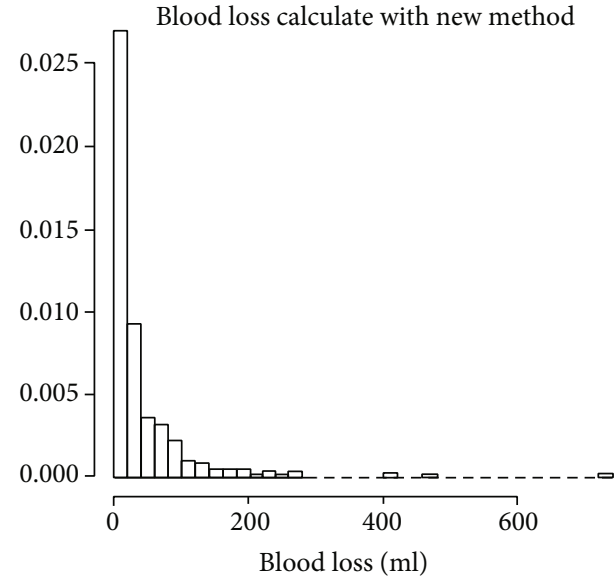

(b)

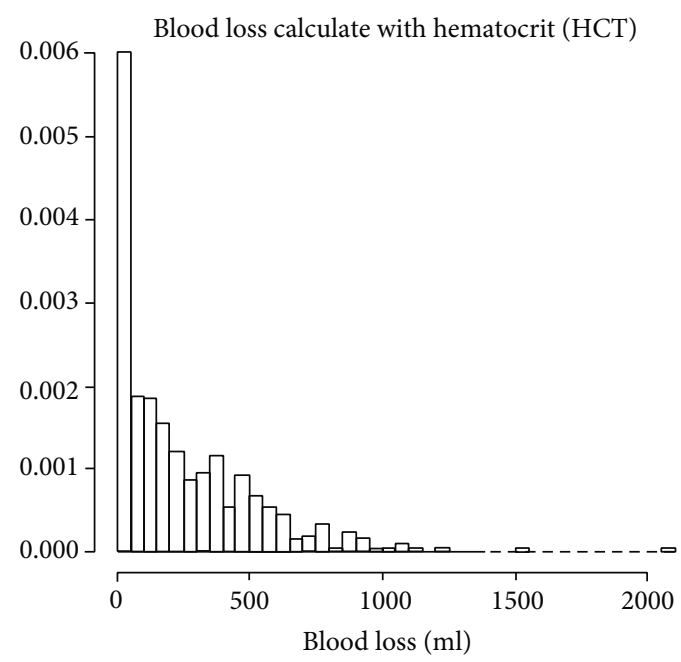

(d)

Figure 2: Blood loss evaluations with three different methods: (a) comparison of blood loss volume (boxplot for mean and variance) in the surgery of TURP with three methods; (b) histogram for blood volume and fitting a density curve with new methods in the process of evaluation blood; (c) histogram for blood volume and fitting a density curve with hemoglobin value in the process of blood evaluation; (d) histogram for blood volume and fitting a density curve with hematocrit value in the process of blood evaluation.

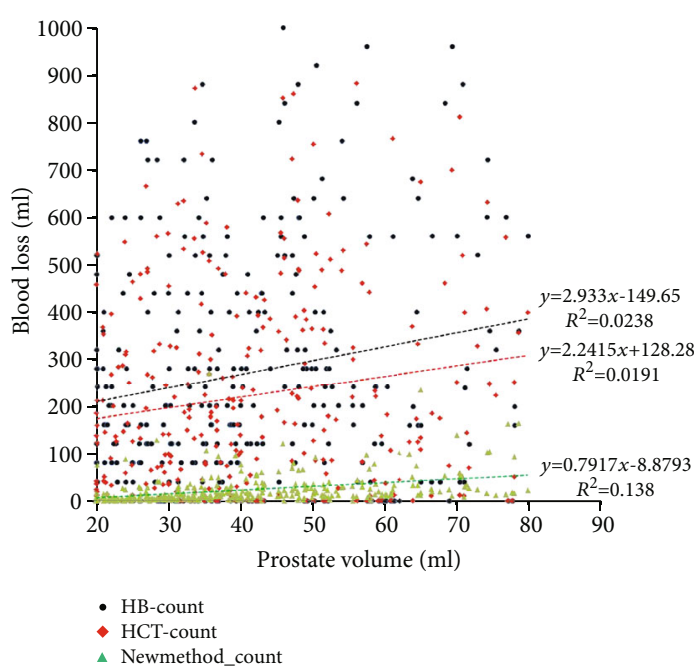

(a)

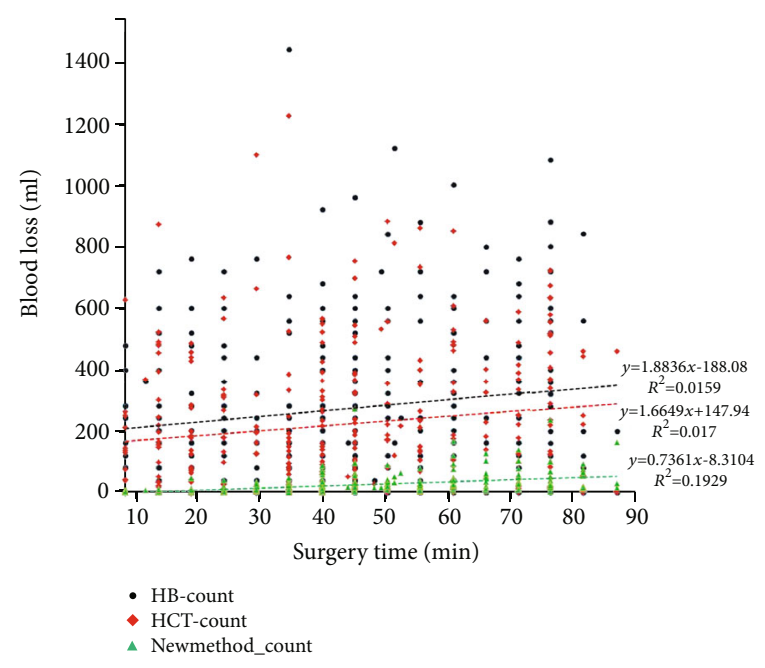

(b)

FIGURE 3: Plot relationships between prostate volume, surgery time, and blood volume with three different methods for evaluating. 
hematocrit percentage was more or equal to preoperative hemoglobin concentration or hematocrit percentage. Blood loss volume calculated with hemoglobin concentration and hematocrit percentage has $20.44 \%$ (65 of 318) and $19.180 \%$ (61 of 318) of 0 value, but not in blood loss calculated with formula (1) (0 of 318, Poisson distribution, $P<0.001$ ). Several reasons can explain this phenomenon. Firstly, a test error was present in clinical lab in the case of a small amount of bleeding [14]. Secondly, the most important reason for hemoglobin or hematocrit percentage rising after surgery was due to different operation lasting times and the patient's fasting time before surgery. In a patient with short surgery time, there is no or a small amount of infusion in TURP, and then, the formation of blood physiological concentration is present after surgery [15].

Among the above various methods mentioned in instruction for determining the amount of bleeding during TURP, HemoCue photometer method, urine-strip method, and pectrophotometer method and the ${ }^{51} \mathrm{Cr}$ isotope labeling method have high accuracy, good repeatability, and simple operation and are worthy of clinical application. However, still, some limitation must be mentioned for the above three methods. Although urine-strip and pectrophotometer methods are low in cost, the reliability of the results needs to be improved mainly due to manual production, measurement, and analysis [9]. The principle of the urine-strip and pectrophotometer method is characterized by heme in $\mathrm{Hb}$ of $\mathrm{RBC}$ having the activity of peroxidase which can catalyze the release of new ecological oxygen by hydrogen peroxide [16]. However, this method will cause technical errors because of the skill problems of the observer during the dilution and interpretation process, resulting in the measured amount of bleeding being 0.5 to 2 times compared with the actual value. With the HemoCue photometer method, it is important to ensure that the RBC is completely hemolyzed in the blood-containing drain to release free $\mathrm{Hb}$ to improve accuracy. Calibration of the instrument is the key in the process [17]. Although the ${ }^{51} \mathrm{Cr}$ isotope labeling method is the most accurate, there are deficiencies in costly, time-consuming, cumbersome instruments and specialized technicians. According to the existing research, there is currently no measurement method that is considered a gold standard [18]. With the development of science and technology and the interdisciplinary application of multiple disciplines, each measurement method will be continuously improved, providing an important research tool for further strengthening intraoperative management, guiding postoperative treatment, and evaluating various new surgical methods. Therefore, bleeding measurement with new developed methods shows accurate, feasible, and cost-effective results. This method for measuring the amount of bleeding in TURP may become an optical choice.

\section{Conclusion}

In conclusion, a new method developed for evaluating bleeding in TURP shows to be accurate, feasible, and cost-effective in clinical application which deserves to be widely used in clinical practice.

\section{Data Availability}

The corresponding data of this manuscript can be available if any researcher required.

\section{Conflicts of Interest}

The authors declare that they have no competing interests.

\section{Authors' Contributions}

XJY was responsible for data collection and data analysis. WY was responsible for data collection. RHW was responsible for data collection and management. LKL was responsible for protocol development. $\mathrm{FH}$ was responsible for project development, manuscript writing, and editing. Xiaojuan Yuan and Wei Yu contributed equally to this work.

\section{Acknowledgments}

The current work was supported partly by the Clinical Medical Research Talents Training Program (2018XLC2017) and partly by the Clinical Major Innovation Feature Technology Project (2018JSLC0021).

\section{References}

[1] P. J. Gilling, N. Barber, M. Bidair et al., "Randomized controlled trial of aquablation versus transurethral resection of the prostate in benign prostatic hyperplasia: one-year outcomes," Urology, vol. 125, pp. 169-173, 2019.

[2] J. Rassweiler, D. Teber, R. Kuntz, and R. Hofmann, "Complications of transurethral resection of the prostate (TURP) - incidence, management, and prevention," European Urology, vol. 50, no. 5, pp. 969-980, 2006.

[3] L. E. Kavanagh, G. S. Jack, and N. Lawrentschuk, "Prevention and management of TURP-related hemorrhage," Nature Reviews Urology, vol. 8, no. 9, pp. 504-514, 2011.

[4] A. Swai, O. V. Nyongole, and A. K. Mteta, "A one year trend of blood loss during transurethral resection of the prostate as seen at urology department, Kilimanjaro Christian Medical Center in Moshi, Tanzania: Do we avoid unnecessary blood transfusion," The Journal of Medical Research, vol. 2, pp. 150-154, 2016.

[5] M. Sarier, I. Duman, S. Kilic et al., "Comparative results of transurethral incision with transurethral resection of the prostate in renal transplant recipients with benign prostate hyperplasia," Urology Journal, vol. 15, no. 4, pp. 209-213, 2018.

[6] P. Bose, F. Regan, and S. Paterson-Brown, "Improving the accuracy of estimated blood loss at obstetric haemorrhage using clinical reconstructions," BJOG: An International Journal of Obstetrics \& Gynaecology, vol. 113, no. 8, pp. 919-924, 2006.

[7] H. Ashraf Aly and H. M. Ramadani, "Assessment of blood loss during cesarean section under general anesthesia and epidural analgesia using different methods," Journal of Anesthesia \& Intensive Care, vol. 9, p. 1, 2006.

[8] J. Ekengren and R. G. Hahn, "Blood loss during transurethral resection of the prostate as measured by the HemoCue photometer," Scandinavian Journal of Urology and Nephrology, vol. 27, no. 4, pp. 501-507, 1993. 
[9] W. Ungjaroenwathana, C. Bunyaratavej, P. Tosukhowong, and T. Dissayabutra, "Estimation of blood loss in transurethral resection of prostate (TUR-P) by urine-strip," Medical journal of the Medical Association of Thailand, vol. 90, no. 11, pp. 2409-2415, 2007.

[10] A. Descazeaud, A. R. Azzousi, C. Ballereau et al., "Blood loss during transurethral resection of the prostate as measured by the chromium-51 method," Journal of Endourology, vol. 24, no. 11, pp. 1813-1816, 2010.

[11] S. Madersbacher, G. Alivizatos, J. Nordling, C. R. Sanz, M. Emberton, and J. J. de la Rosette, "EAU 2004 guidelines on assessment, therapy and follow-up of men with lower urinary tract symptoms suggestive of benign prostatic obstruction (BPH guidelines)," European Urology, vol. 46, no. 5, pp. 547-554, 2004.

[12] T. C. McCullough, J. V. Roth, P. C. Ginsberg, and R. C. Harkaway, "Estimated blood loss underestimates calculated blood loss during radical retropubic prostatectomy," Urologia Internationalis, vol. 72, no. 1, pp. 13-16, 2004.

[13] M. Brecher, T. Monk, and L. Goodnough, "A standardized method for calculating blood loss," Transfusion, vol. 37, no. 10, pp. 1070-1074, 1997.

[14] M. Plebani, "Errors in clinical laboratories or errors in laboratory medicine?," Clinical Chemistry and Laboratory Medicine (CCLM), vol. 44, no. 6, pp. 750-759, 2006.

[15] D. Zindrou, K. M. Taylor, and J. P. Bagger, "Preoperative haemoglobin concentration and mortality rate after coronary artery bypass surgery," The Lancet, vol. 359, no. 9319, pp. 1747-1748, 2002.

[16] N. Ersoy, G. Ersoy, and M. Kutlu, "Assessment of hydration status of elite young male soccer players with different methods and new approach method of substitute urine strip," Journal of the International Society of Sports Nutrition, vol. 13, no. 1, p. 34, 2016.

[17] R. Hiscock, D. Kumar, and S. Simmons, "Systematic review and meta-analysis of method comparison studies of Masimo pulse co-oximeters (Radical- $7^{\mathrm{TM}}$ or Pronto- $7^{\mathrm{TM}}$ ) and Hemo Cue ${ }^{\circledR}$ absorption spectrometers (B-Hemoglobin or 201+) with laboratory haemoglobin estimation," Anaesthesia and Intensive Care, vol. 43, pp. 341-350, 2015.

[18] P. Mollison and N. Veall, "The use of the isotope $51 \mathrm{Cr}$ as a label for red cells," British Journal of Haematology, vol. 1, no. 1, pp. 62-74, 1955. 